\title{
Atmosphärische Deposition und Anreicherung von Schwermetallen und Stickstoff in Natura-2000-Gebieten Deutschlands
}

\author{
Winfried Schröder • Roland Pesch • Harald Zechmeister • Werner Kratz • Marcel Holy • Harry Harmens • \\ Hilde Fagerli • Ilia Ilyin
}

Erhalten: 26. Februar 2010/Akzeptiert: 7. Juni 2010/Online veröffentlicht: 24. Juni 2010

(C) Springer-Verlag 2010

Zusammenfassung Absicht Im Rahmen der Luftreinhaltekonvention werden Moose zur europaweiten Kartierung der Bioakkumulation von Schwermetallen und Stickstoff verwendet. Hierzu werden alle fünf Jahre Moose gesammelt und chemisch analysiert. Diese Daten werden in dem vorliegenden Artikel für die Natura-2000-Gebiete Deutschlands (SCI) ausgewertet.

Methoden Die zeitlichen Trends der Metallanreicherungen in den SCIs wurden anhand eines Multimetallindex berechnet, der mit Verfahren der Geostatistik und der Perzentilstatistik abgeleitet wurde. Für die Stickstoffbioakkumulation lagen Messdaten aus dem Jahr 2005 vor. Aus den Messdaten der Metall- und Stickstoffanreicherung wurden mittels linearer Regressionsanalyse Depositionswerte be-

W. Schröder $\cdot$ R. Pesch $(\varangle) \cdot$ W. Kratz $\cdot$ M. Holy

Lehrstuhl für Landschaftsökologie, Hochschule Vechta,

PF 1553, 49364 Vechta, Deutschland

E-Mail: rpesch@iuw.uni-vechta.de

H. Zechmeister

Universität Wien,

Althanstraße 14, 1090 Wien, Österreich

E-Mail: Harald.Zechmeister@univie.ac.at

H. Harmens

Centre for Ecology and Hydrology,

Bangor, Gwynedd LL57 2UW, UK

E-Mail: hh@ceh.ac.uk

H. Fagerli

Research and Development Department,

Norwegian Meteorological Institute,

Oslo, Norway

E-Mail: h.fagerli@met.no

I. Ilyin

Meteorological Synthesizing Centre East of EMEP,

Krasina pereulok, 16/1, 123056 Moscow, Russia

E-Mail: ilia.ilyin@msceast.org rechnet. Diese wurden dann für die SCIs in Deutschland ausgewertet.

Ergebnisse Die höchsten atmosphärischen Metalleinträge wurden für 1990 berechnet. Anschließend nahmen diese deutlich bis zum Jahr 2000 ab, um dann wieder bis 2005 anzusteigen. Für Stickstoff wurde ein Median von 1,5\% in der Moostrockenmasse ermittelt. Die aus den Daten zur Bioakkumulation berechneten Depositionswerte belaufen sich auf $0,33 \mathrm{~g} / \mathrm{ha} / \mathrm{a}$ für Cadmium, 8,2 $\mathrm{g} / \mathrm{ha} / \mathrm{a}$ für Blei und $16,7 \mathrm{~kg} /$ ha/a für Stickstoff.

Schlussfolgerungen Das Moosmonitoring ist das einzige Umweltbeobachtungsprogramm, mit dem die atmosphärische Schwermetall- und Stickstoffexposition der deutschen Natura2000-Gebiete statistisch begründet geschätzt werden kann.

Schlüsselwörter Bioakkumulation · Deposition · Moose · Natura-2000-Gebiete (SCI) - Schwermetalle $\cdot$ Stickstoff

\section{Atmospheric Deposition and Accumulation of Heavy Metals and Nitrogen in Natura 2000 sites in Germany}

Abstract Purpose Under the Convention on Long-range Transboundary Air Pollution mosses are used to map the bioaccumulation of heavy metals and nitrogen throughout Europe. To this end, since 1990 mosses were sampled and analyzed chemically every five years. The goal of this article is to apply the moss survey data for assessing the bioaccumulation of $\mathrm{Cd}, \mathrm{Pb}$ and $\mathrm{N}$ in German Natura 2000 Sites of Community Importance (SCI).

Methods The temporal trends of the heavy metal bioaccumulation within SCIs were analyzed using a multi metal index (MMI) calculated by means of geostatistics and percentile statistics. For nitrogen, only monitoring values for 2005 were available for the assessment. The geostatistically 
estimated values of the metal and nitrogen concentrations in mosses were transformed to estimated deposition values by use of regression analyses. Subsequently, the results were integrated into the assessment of the German SCIs.

Results Highest metal loads within SCIs were detected in 1990, followed by a continuous decrease to 2000 and a significant increase until 2005. Regarding $\mathrm{N}$, a median of $1.5 \%$ nitrogen in the dry mass was calculated. The deposition values calculated from the moss estimates resulted in median values of $0.33 \mathrm{~g} / \mathrm{ha} / \mathrm{a}$ for $\mathrm{Cd}, 8.2 \mathrm{~g} / \mathrm{ha} / \mathrm{a}$ for $\mathrm{Pb}$ and $16.7 \mathrm{~kg} / \mathrm{ha} / \mathrm{a}$ for nitrogen.

Conclusions The Moss-Monitoring is the only environmental monitoring programme which enables statistically sound estimations of the exposure of SCI to environmental contaminants in terms of heavy metal and nitrogen deposition and bioaccumulation.

Keywords Bioaccumulation - Deposition · Heavy Metals · Mosses · Natura 2000 Sites of Community Importance $(\mathrm{SCI}) \cdot$ Nitrogen

\section{Hintergrund und Ziel}

In die Atmosphäre emittierte Stoffe wie die in diesem Artikel behandelten Schwermetalle Cadmium $(\mathrm{Cd})$ und Blei $(\mathrm{Pb})$ sowie Stickstoff $(\mathrm{N})$ gelangen nach ihrer atmosphärischen Ausbreitung mit Regen und Schnee als nasse Depositionen, mit Wolken bzw. Nebel als okkulte (feuchte) Deposition und partikulär oder gasförmig als trockene Deposition auf die Erdoberfläche (Slanina und Wayne 2008). Dort können sich die Metalle und der Stickstoff anreichern und ökologische Veränderungen bewirken: N-Verbindungen tragen zur Eutrophierung und Versauerung terrestrischer und aquatischer Ökosysteme bei und können wie auch über toxische Wirkungen von $\mathrm{Cd}$ und $\mathrm{Pb}$ die Biodiversität beeinflussen (Ahlf et al. 2009; Erisman und de Vries 2000; Förstner 2009; Galloway et al. 2008; Gundersen und Rasmussen 1990; Lorenz und Granke 2009; MERAG 2007; Pitcairn et al. 1998, 2006; Selck und Forbes 2004; Solga et al. 2005; Worms et al. 2006). Um dies zu verhindern, werden umweltpolitische Maßnahmen zur Reduktion der N-Emissionen ergriffen. In Deutschland sind dies beispielsweise die Biodiversitätsstrategie (Mohaupt-Jahr und Küchler-Krischun 2008) sowie das Stickstoffminderungsprogramm (UBA 2008) und auf europäischer Ebene die Genfer Luftreinhaltekonvention (Convention on Long-range Transboundary Air Pollution, CLRTAP) (UNECE 1979).

Die Europäische Kommission entwickelte Vorgaben zur Auswertung von Umweltdaten, verbunden mit Forderungen nach einer integrierten Umweltbeobachtung und -bewertung, begleitet von Informationsanforderungen der EUGesetzgebung wie z.B. die Fauna-Flora-Habitat-Richtlinie (FFH-RL, 92/43/EWG) und die Wasserrahmen-Richtlinie
(WRRL, 2000/60/EG), (Doyle und Heiß 2009). Zum Schutz von Pflanzen- und Tierarten sowie ihrer natürlichen Lebensräume werden europaweit durch die Mitgliedstaaten Gebiete von gemeinschaftlicher Bedeutung (Sites of Community Importance, SCI) nach Maßgaben der Richtlinie 92/43/EWG (FFH-Richtlinie) eingerichtet. Zusammen mit den auf Grundlage der Richtlinie 79/409/EWG (Vogelschutzrichtlinie) ausgewiesenen Vogelschutzgebieten (Special Protection Areas, SPA) bilden diese das Natura-2000-Schutzgebietsnetzwerk. Die FFH-Richtlinie verpflichtet zur Durchführung eines allgemeinen Monitorings des Erhaltungszustandes der Arten und Lebensraumtypen von gemeinschaftlichem Interesse (Doyle und Heiß 2009). Schad- und Nährstoffeinträge in Natura-2000-Gebiete können dort in aquatischen und in terrestrischen Ökosystemen deren ökologischen Zustand verschlechtern oder das Erreichen der für ein Natura-2000Gebiet definierten Ziele verhindern. Zur Abschätzung möglicher Schadwirkungen auf Arten und Lebensraumtypen durch Stoffeinträge ist zu klären, um welche Stoffe es sich handelt und in welcher Menge pro Raum- und Zeiteinheit die zu schützenden Arten und Lebensräume ihnen ausgesetzt sind. Daten über diese Belastungen sind mit Daten über Wirkschwellen, also denjenigen Stoffkonzentrationen, die bei den zu schützenden Arten und Biotopen erhebliche ökologische Veränderungen und damit ein Nichterreichen der Schutzziele bewirken, abzugleichen. Zur Abschätzung der stofflichen Exposition in Natura-2000-Gebieten werden vielfach die modellierten Depositionen verwendet, deren methodische Harmonisierung und räumliche Repräsentanz nicht unproblematisch sind (Gauger 2005; Gauger und Anshelm 2002; Gauger et al. 2000a,b, 2002, 2008a,b; Schröder et al. 2010a,b). Hinzu kommt, dass die aktuellsten Werte der modellierten Depositionen von Schwefel und Stickstoff um 45 bzw. $35 \%$ und die der Metalle Cadmium und Blei um mindestens $200 \%$ niedriger als die bislang veröffentlichten Daten liegen. Grund hierfür ist eine veränderte Modellierungstechnik (UBA 2010). Folglich können die gebietsspezifischen Gesamtbelastungen oft entweder überhaupt nicht oder nur unter Vorbehalt auf Basis dieser Datengrundlage abgeschätzt werden. Die wirkungsseitige Beurteilung der Erheblichkeit möglicher Beeinträchtigungen von Arten bzw. Lebensraumtypen durch Stoffeinträge müsste anhand von Daten über Wirkschwellen für schutzwürdige Arten und Lebensräume (Ökosysteme) erfolgen, die jedoch weitgehend fehlen. Auf Ökosystemebene stehen für einige Stoffe Critical Levels/Loads zur Verfügung, deren Über- oder Unterschreitung jedoch wiederum anhand der modellierten Depositionen festgestellt wird. Wegen dieser unzureichenden Verfügbarkeit von Expositions- und Wirkungsdaten ist eine fachgerechte Beurteilung von Stoffeinträgen in Natura-2000-Gebiete derzeit nur sehr eingeschränkt möglich (Doyle und Heiß 2009). Eine Verbesserung dieser Situation ließe sich dadurch erreichen, dass man die modellierten Daten über die atmosphärischen Depositionen 
mit geeigneten Messdaten über ökosystemare Wirkungsschwellen abgleicht und auf Plausibilität überprüft. Hierfür sollten Daten aus komplementären methodischen Ansätzen Depositionsmessungen und -modellierungen einerseits und Moosmonitoring andererseits - für das Gebiet Deutschlands miteinander verknüpft werden, so wie es europaweit bereits erfolgt ist (Schröder 2010a,b).

Daten über Emissionsquellen, atmosphärische Einträge (Depositionen) und Anreicherungen (Akkumulationen) von Stoffen in der Umwelt beschreiben miteinander verknüpfte Vorgänge, die zusammen zur Identifikation von Prioritäten und zur Überprüfung der Effektivität umweltpolitischer Maßnahmen benötigt werden. Das Pollutant Release and Transfer Register (PRTR; BMU 2006) enthält Emissionsdaten zu 91 Schadstoffen (EG/166/2006). Die OSPARVertragsstaaten messen 42 persistente, bioakkumulierende und toxische (PBT-)Stoffe im Nordostatlantik, um die Maßnahmen zur Reduzierung der Stoffbelastungen zu überwachen (OSPAR 2007). Eine flächendeckende Erfassung von Schadstoffbelastungen terrestrischer Ökosysteme erfolgt z. B. für Schwermetalle und Stickstoff im Moosmonitoring (Harmens et al. 2008, 2009) auf Grundlage der Genfer Luftreinhaltekonvention. Die in Moosen gemessenen Schwermetall- und Stickstoffgehalte sind mit den entsprechenden atmosphärischen Depositionen statistisch korreliert (Mohr et al. 2009; Schröder et al. 2010a,b), da Moose nasse, okkulte und trockene Depositionen sammeln (Knappe et al. 2008), deren relative Anteile an den Akkumulationen in Moosen bislang experimentell nicht systematisch bestimmt wurden. Das Moosmonitoring ermöglicht aufgrund seiner international harmonisierten Methodik, der umfassenden Qualitätssicherung im Sinne von O'Connor (2009) sowie der hohen räumliche Auflösung eine wissenschaftlich fundierte Maßnahmenplanung, die den jeweiligen regionalen Verhältnissen Rechnung trägt und dabei gleichermaßen den Informationsbedarf für die nationale und internationale Depositionsmodellierung und die Erfolgskontrolle erfüllt (Doyle und Heiß 2009).

Die deutsche Biodiversitätsstrategie sieht vor, das Genfer Luftreinhalteabkommen (UNECE 1979) weiterzuentwickeln, das am Vorsorgeprinzip orientierte Chemikalienmanagement zu unterstützen und das UNECE Multikomponenten-Protokoll umzusetzen (BMU 2007, S. 55, 80). Gemäß der Stickstoffminderungsstrategie des Bundes kommt es bei der Bewertung der Wirkungen diffuser Stoffeinträge wesentlich darauf an, die räumliche Differenzierung der Stickstoffanreicherungen in Schutzgütern wie der Vegetation zu erfassen und die bisherigen Schätzungen ,durch Monitoring oder andere Verbesserungen der Methoden und Daten zu validieren“ (UBA 2008, S. 48). Diesem Aspekt war der European Cooperation in Science and Technology Workshop Nitrogen and Natura 2000 (18. bis 20. Mai 2009, Brüssel) gewidmet (Whitfield und Strachan 2009). Ziel war es, bewährte Ansätze zur Ermittlung atmosphärischer Stickstoffeinträge in Natura-2000-Schutzgebiete im Hinblick auf umweltpolitische Handlungsoptionen zu analysieren und zu bewerten. Der Anlass des Workshops bezieht sich auf die Verpflichtung der EU-Mitgliedstaaten zur Erhaltung der Biodiversität (RL 92/43/EWG), da neben Schwermetalleinträgen auch hohe Stickstoffeinträge mit einer Verminderung der Artenvielfalt einhergehen. Gesucht wurde ein wissenschaftlich anerkannter europaweiter Ansatz, mit dem sich Auswirkungen von Stickstoffanreicherungen auf Natura2000-Gebieten grenzüberschreitend ermitteln lassen. Wissenschaftler sowie Vertreter der EU-Mitgliedsstaaten und der EU-Kommission empfehlen den EU-Mitgliedsstaaten eine enge Zusammenarbeit mit dem ICP Vegetation, um Moose als Indikatoren der Stickstoffanreicherung in Natura 2000 Sites of Community Importance (SCI) zu verwenden (Whitfield und Strachan 2009). Die Anreicherung von Stickstoff in biologischem Material sei der erste Schritt zu einer Wirkung und ermögliche so das frühzeitige Erkennen potenzieller Spätfolgen (Eutrophierung, Versauerung, Verringerung der Biodiversität). Dies sei wichtig, da momentan zu beobachtende Veränderungen in terrestrischen Ökosystemen nicht nur auf derzeitige, sondern auch auf ehemals hohe Stickstoffeinträge zurückzuführen seien. Die Eignung von Moosen zur Erfassung atmosphärischen Schwermetall- und Stickstoffeintrags wird als nachgewiesen anerkannt (Zechmeister et al. 2003). In Natura 2000 SCI einiger Mitgliedsstaaten (z. B. Dänemark) finden Moose als Anreicherungsindikatoren Verwendung. Der vorliegende Artikel beinhaltet den Beitrag der Verfasser zu dem genannten COST-Workshop. Im Folgenden wird aufgezeigt, wie auf Grundlage des Moosmonitorings die Schwermetall- und Stickstoffexposition von $3492^{1}$ FFH-Gebieten in Deutschland geschätzt wurde. Hierbei wird die Exposition in atmosphärische Deposition und Bioakkumulation differenziert.

\section{Material und Methoden}

\subsection{Grundlagen des Moosmonitorings}

Die Methodik des Moosmonitorings wird hier nur sehr grob skizziert, denn dieser Artikel ist Teil einer Beitragsserie, in der die methodischen Details in einem eigenen Beitrag behandelt werden (Schröder et al. 2009). Moose reichern über mehrere Jahre atmosphärische Depositionen von Schwermetallen und Stickstoff ohne physiologische Beeinträchtigung an. Deswegen sind sie für das Monitoring der Bioakkumulation atmosphärischer Depositionen - für anlagenbezogenes

\footnotetext{
${ }^{1}$ Dies entspricht nicht dem aktuellen Stand der FFH-Gebiete in Deutschland, da für die Berechnungen nur ein aus dem Jahr 2002 stammender GIS-Datensatz zur Verfügung stand.
} 
Monitoring ebenso wie für regionale, nationale und kontinentale Surveys - besonders geeignet (Forster et al. 1993; Genßler et al. 2001; Herpin et al. 2004; Kostka-Rick et al. 2001; Mohr 1999, 2007; Mohr et al. 2009; Pesch et al. 2008; Schröder und Pesch 2005; Schröder et al. 2008a,b, 2009; Suchara et al. 2007; Sucharová et al. 2008; Wappelhorst et al. 2000; Zechmeister et al. 2006). In Europa wird das Moosmonitoring seit 1990 alle fünf Jahre durchgeführt (Harmens et al. 2008). Ziel ist es, die räumlichen Muster und zeitlichen Entwicklungen der Metall- und Stickstoffbioakkumulation staatenübergreifend zu kartieren. Dabei geht es nicht um die Erfassung von Stoffgehalten in Moosen, die in der Nachbarschaft von Emittenten gesammelt wurden, sondern um sogenannte Hintergrundwerte an Orten ohne erkennbaren Emittenteneinfluss. Im Moosmonitoring werden Waldgebiete beprobt, wobei von potenziellen Emissionsquellen bestimmte Mindestabstände einzuhalten sind.

In Deutschland führten Bund und Länder das Moosmonitoring in den Jahren 1990, 1995, 2000 und 2005 gemeinsam durch. Die räumliche Dichte des Moosmessnetzes betrug 1,7 (1990), 2,9 (1995, 2000) und 2,0 (2005) Standorte pro $1000 \mathrm{~km}^{2}$. Europaweit kommen auf $1000 \mathrm{~km}^{2}$ mindestens 1,5 Moosentnahmestellen. Die Entnahme und die Analyse der Moosproben erfolgten nach einer europaweit verwendeten Richtlinie (UNECE/CLRTAP 2005). Die Qualität der Datengewinnung wird von ihrer Planung bis zum Datenmanagement umfassend im Sinne von O'Connor (2009) gesichert und dokumentiert (Harmens et al. 2008; Schröder et al. 2009).

\subsection{Flächenhafte Schätzung der Schwermetall-} und Stickstoffanreicherung

Da das Moosmessnetz nicht vollständig deckungsgleich mit der geografischen Verteilung der FFH-Gebiete ist, wurde die Abschätzung der Stoffakkumulation auf Basis von geostatistisch geschätzten Flächeninformationen durchgeführt. So lag die Anzahl an Moosmonitoringstandorten innerhalb von FFH-Gebieten (Stand 2002) 1990 bei 86, 1995 bei 112, 2000 bei 111 und 2005 bei 83 Standorten. Puffert man die FFH-Gebiete mit $2 \mathrm{~km}$, steigt die Anzahl der innerhalb und im Umfeld der FFH-Gebiete beprobten Standorte auf 308 (1990), 526 (1995), 532 (2000) und 391 (2005). Dies entspricht einem prozentualen Anteil von mindestens $50 \%$ SCI im Moosmonitoringmessnetz pro Kampagne.

Für die Einschätzung der in den Moosen akkumulierten Elementgehalte innerhalb der FFH-Gebiete wurden die in Deutschland gemessenen Anreicherungen der Metalle Cr, $\mathrm{Cu}, \mathrm{Fe}, \mathrm{Ni}, \mathrm{Pb}, \mathrm{Ti}, \mathrm{V}$ und $\mathrm{Zn}$ sowie $\mathrm{N}$ in Moosen zunächst jeweils auf räumliche Autokorrelation überprüft (Variogrammanalyse). Die für die genannten Elemente bestimmten Variogrammfunktionen wurden anschließend für die Schätzung der Bioakkumulation in einem $3 \times 3 \mathrm{~km}^{2}$-Raster verwendet. Die resultierenden GIS-Karten zeigen die räum- liche Differenzierung der Metallakkumulation von 1990 bis 2005 und bilden die Grundlage für die Berechnung des Multimetallindex (MMI). Bezogen auf die Stickstoffanreicherungen in den Moosen erfolgte die Berechnung von Flächenkarten sowohl für die Stickstoffmessdaten wie auch für solche, die daraus anhand der Schwefelkonzentration nach Mohr et al. (2009) berechnet wurden. ${ }^{2}$ Basierend auf den Ergebnissen einer europaweiten Auswertung wurden ferner geostatistisch berechnete Karten der $\mathrm{Cd}$-, $\mathrm{Pb}$ - und N-Akkumulation in einer Auflösung von $5 \times 5 \mathrm{~km}^{2}$ für die Schätzung atmosphärischer Depositionsraten verwendet.

Die Multimetallindizes wurden auf Grundlage der Perzentilstatistik der Metallbioakkumulation berechnet. Ein Perzentil ist derjenige Wert einer Messgröße, der von einem bestimmten Prozentanteil der Messwerte unterschritten wird. Das 20. Perzentil ist also derjenige Messwert in einer von niedrigen zu hohen Werten geordneten Messwertreihe, der von $20 \%$ der Messwerte unterschritten wird. Der MMI wurde sowohl für die Messwerte als auch für die aus ihnen berechneten flächenhaften Schätzwerte ermittelt. Dazu wurden vorerst pro Element 10 Perzentilklassen gebildet, sodass jedem Standort bzw. jeder Rasterzelle ein elementspezifischer Indexwert zwischen 1 (niedrige Bioakkumulation) und 10 (hohe Bioakkumulation) zugewiesen werden konnte. Der $\mathrm{MMI}_{1990-2005}$ eines jeden Moossammelortes oder einer jeden Rasterzelle der geostatistisch berechneten Flächenkarten entspricht dem Durchschnitt der elementspezifischen Indexwerte der Konzentrationen von $\mathrm{Cr}, \mathrm{Cu}, \mathrm{Fe}, \mathrm{Ni}$, $\mathrm{Pb}$, V, Ti und $\mathrm{Zn}$, die durchgängig 1990, 1995, 2000 und 2005 in Deutschland gemessen wurden. Die Karten der regionalen Differenzierung des $\mathrm{MMI}_{1990-2005}$ wie auch der geostatistischen Karten zu der Stickstoffanreicherung wurden abschließend mit dem Natura 2000 SCI Polygon Layer Deutschlands verschnitten und statistisch ausgewertet.

\subsection{Flächenhafte Schätzung der atmosphärischen} Depositionen von Blei, Cadmium und Stickstoff

Die geostatistisch berechneten Flächenkarten der europaweiten $\mathrm{Cd}-, \mathrm{Pb}-$ und $\mathrm{N}$-Anreicherung in Moosen (2005) (Abschn. 2.2) wurden mit Karten der Cd-, Pb- und N-Gesamtdeposition (2005) aus dem European Monitoring and Evaluation Program (EMEP) der United Nations Economic Commission for Europe (UNECE) im GIS verschnitten. Anschließend wurden die Mediane aller $5 \mathrm{~km}^{2}$-Rasterzellen der $\mathrm{Cd}$-, $\mathrm{Pb}$ - und $\mathrm{N}$-Anreicherungskarten innerhalb der je-

\footnotetext{
${ }^{2}$ Dieses Vorgehen ist dadurch begründet, dass die Schwefelkonzentrationen in Moosen bei den aktuell sehr niedrigen $\mathrm{SO}_{2}$-Immissionen großräumig nur gering variieren (Mohr 1999). Unterschiede der SGehalte sind somit in großen Teilen Deutschlands vorrangig auf abweichende Depositionsbedingungen am Probenentnahmeort zurückzuführen, die z.B. durch die vertikale und horizontale Struktur der Vegetation hervorgerufen werden können.
} 
weiligen $50 \times 50 \mathrm{~km}^{2}$-EMEP-Rasterzellen berechnet. Die Mediane der geschätzten Elementkonzentrationen im Moos wurden dann zusammen mit den EMEP-Depositionswerten ln-transformiert und korrelations- und regressionsanalytisch untersucht. Die aus den Analysen resultierenden Regressionsfunktionen wurden auf die Kriging-Flächenkarten der Stoffanreicherungen in Moosen zur Ableitung von Regressionskarten für $\mathrm{Cd}, \mathrm{Pb}$ und $\mathrm{N}$ angewendet. Die Residuen der Regressionsanalysen entsprechen dem Anteil der unerklärten Varianz der abhängigen Variablen (hier der EMEPDepositionen). Geostatistisch betrachtet quantifizieren sie die Abweichungen zu räumlich variierenden Erwartungswerten einer Zufallsfunktion auf, die über die Regressionskarten abgebildet werden. Die Residuen wurden daher entlogarithmiert, auf räumliche Autokorrelation untersucht und mit Lognormal-Kriging flächenhaft interpoliert. Zur Berechnung der Gesamtdepositionskarten wurden die Residuenkarten mit den regressionsanalytisch berechneten Depositionsflächenkarten verrechnet. Diese Europakarten der $\mathrm{Cd}$-, Pb- und N-Gesamtdeposition des Jahres 2005 wurden abschließend mit dem Natura 2000 SCI Polygon Layer Deutschlands verschnitten und statistisch ausgewertet.

\section{Ergebnisse}

Die Regressionsanalysen zeigen, dass die N-Anreicherung in den Moosen aus Hintergrundgebieten mit der N-Gesamtdeposition europaweit mit $r_{p}=0,79$ bzw. $r_{s}=0,70$ korreliert ist (Schröder et al. 2010a). Das Bestimmtheitsmaß des Regressionsmodells beträgt $R^{2}=62,81$. Die statistische Auswertung der auf dieser Grundlage berechneten Karte der N-Gesamtdeposition ergibt einen deutschlandweiten Mittelwert von $18,1 \mathrm{~kg} / \mathrm{ha} / \mathrm{a}$ (Standardabweichung 3,49 kg/ha/a). Die Vegetationsbedingungen der Probenentnahmestandorte im Moosmonitoring entsprechen annähernd der von Gauger et al. (2008a) u. a. berücksichtigten seminatürlichen Vegetation. Für diesen Rezeptortyp errechnet sich aus den Daten von Gauger et al. (2008a) ein bundesweiter Mittelwert von $21,47 \mathrm{~kg} / \mathrm{ha} / \mathrm{a}$. Der anhand des Moosmonitorings berechnete Mittelwert (18,1 kg/ha/a) ist um rund $34 \%$ niedriger als das von Gauger et al. (2008a) über alle unterschiedlichen Vegetationstypen berechnete bundesweite Mittel von 27,4 kg/ ha/a. Die am Ende des Jahres 2009 anlässlich eines Workshops zur Modellierung von Schadstoffeinträgen und ihren Wirkungen auf Ökosysteme veröffentlichten N-Gesamtdepositionsmodellierungen liegen um durchschnittlich $35 \%$ (UBA 2010) niedriger und würden damit denen entsprechen, die anhand der Daten aus dem EMEP und ICP Vegetation in dieser Untersuchung berechnet wurden. Die Regressionsmodelle zeigen ferner, dass die Cd-Anreicherungen in den Moosen mit der Cd-Gesamtdeposition europaweit hoch korreliert sind $\left(r_{p}=0,67, r_{s}=0,69\right)$. Das Bestimmtheitsmaß des Regressionsmodells beträgt $R^{2}=44,23$ (Schröder et al. 2010b). Die statistische Auswertung der auf Grundlage dieses Modells berechneten Depositionskarte ergibt, dass die Cd-Gesamtdeposition im deutschlandweiten Mittel der Jahre 2003 bis 2005 0,342 g/ha/a (Standardabweichung 0,08 g/ ha/a) betrug. Die Pb-Akkumulationen in den Moosen korrelieren mit der Pb-Gesamtdeposition deutlich $\left(r_{p}=0,76, r_{s}=\right.$ 0,77). Das Bestimmtheitsmaß des Regressionsmodells beträgt $R^{2}=57,87$ (Schröder et al. 2010b). Die Auswertung der aus diesem Modell berechneten Pb-Depositionskarte ergibt, dass die bundesweit durchschnittliche Pb-Gesamtdeposition im Jahr 2005 8,6 g/ha/a (Standardabweichung 2,1 g/ha/a) betrug. Die Cd-Depositionen liegen zwischen den Berechnungen von Knappe et al. (2008) (0,86 g/ha/a Cd-Gesamtdeposition in ländlichen Gebieten) und denen von Gauger et al. (2008) (0,01-0,13 g/ha/a). Die aus dieser Untersuchung hervorgegangene Schätzung der $\mathrm{Pb}$-Deposition liegt ebenfalls zwischen den Werten von Gauger et al. (2008) (3,56-4,46 g/ ha/a) und Knappe et al. (2008) (28,7 g/ha/a).

Die Ergebnisse der Verschneidung der FFH-Gebiete Deutschlands mit den Karten über den $\mathrm{MMI}_{1990-2005}$ ist in der Abb. 1 dargestellt. Diese zeigt, dass der bundesweite Median des $\mathrm{MMI}_{1990-2005}$ innerhalb von FFH-Gebieten von 1990 $\left(\mathrm{MMI}_{\mathrm{Med}}=8,0\right)$, über $1995\left(\mathrm{MMI}_{\mathrm{Med}}=6,6\right)$ bis $2000\left(\mathrm{MMI}_{\mathrm{Med}}\right.$ $=3,3)$ kontinuierlich abnimmt. Von 2000 bis 2005 ist allerdings ein signifikanter Anstieg des $\mathrm{MMI}_{1990-2005}$-Medians auf 4,1 festzustellen. Die Stickstoffanreicherung in den FFH-Gebieten (nicht abgebildet) zeigt im Hinblick auf die originalen geschätzten Werte einen bundesweiten Median von 1,5\% N in der Trockenmasse; für die an der Schwefelkonzentration in den Moosen standardisierten Schätzungen lässt sich ein Median von 1,3\% N in der Trockenmasse festhalten.

Die FFH-spezifische Auswertung der auf Basis von Moosakkumulationen berechneten Depositionskarten zeigt die Abb. 2. Bezogen auf das gesamte Bundesgebiet beträgt der Median der Cd-Depositionen $0,33 \mathrm{~g} / \mathrm{ha} / \mathrm{a}$, wobei mit $0,81 \mathrm{~g} /$ ha/a die höchsten Cd-Depositionen für die FFH-Gebiete $B \ddot{a}$ renstein, Hammerberg, Schlangenberg, Steinbruchbereich Bernhardshammer/Binsfeldhammer, Wehebachtäler und Leyberg sowie Werther Heide geschätzt wurden. Die Pb-Depositionen zeigen im bundesweiten Schnitt 8,2 $\mathrm{g} / \mathrm{ha} / \mathrm{a}$ mit maximalen Werten von $20 \mathrm{~g} / \mathrm{ha} / \mathrm{a}$ in dem Gebiet Wurmtal südlich Herzogenrath, und die Stickstoffeinträge weisen bundesweit betrachtet einen Median von $16,7 \mathrm{~kg} / \mathrm{ha} / \mathrm{a}$ auf. Maximale N-Depositionen finden sich im FFH-Gebiet Emsaue, Kreise Warendorf und Gütersloh mit 29,4 kg/ha/a. Alle Maxima befinden sich in FFH-Gebieten Nordrhein-Westfalens.

\section{Diskussion und Schlussfolgerungen}

Die Exposition eines Organismus lässt sich differenzieren in die äußere und innere Exposition. Letztere stellt die Kon- 

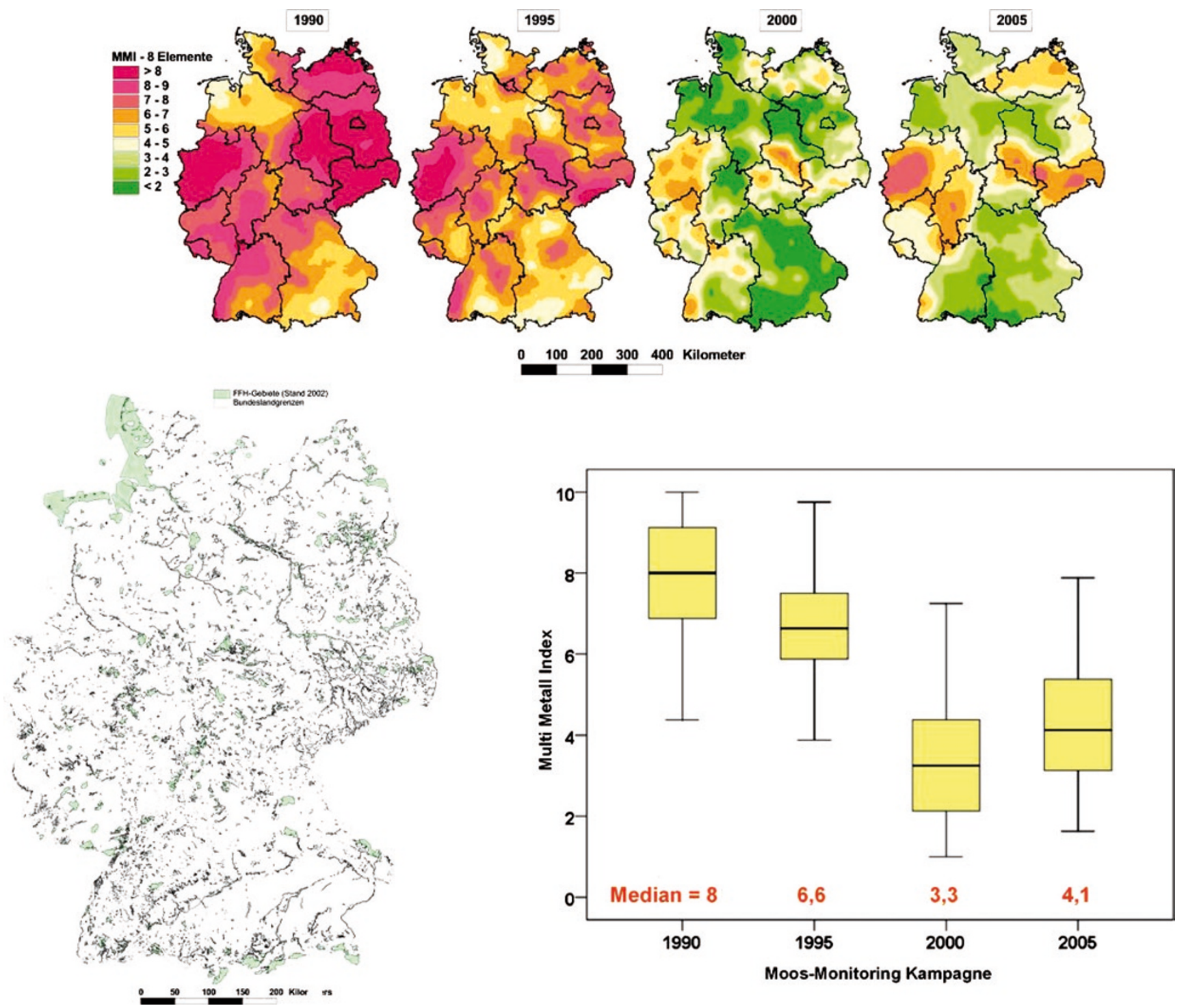

Abb. 1 Ausprägung des perzentilbasierten Multimetallindexes für die Metallbioakkumulation zwischen 1990 und 2005 (MMI $1990-2005)$ innerhalb von FFH-Gebieten
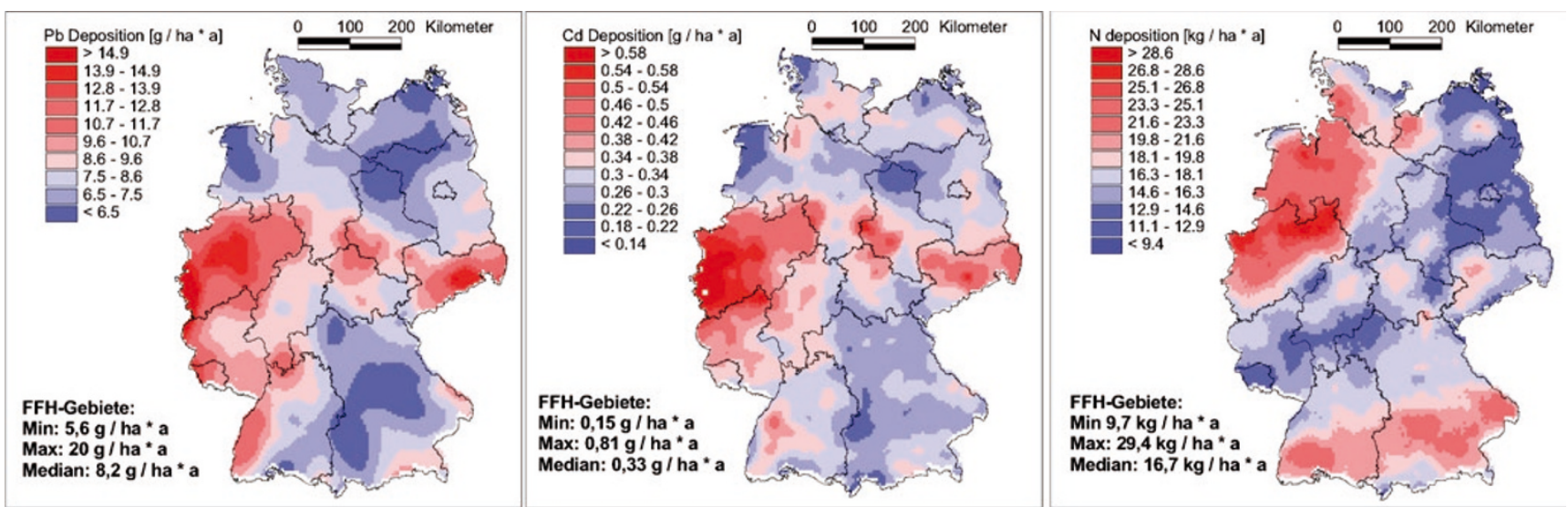

Abb. 2 Auf Basis der Stoffakkumulation in Moosen berechnete Cd-, Pb- und Gesamtstickstoffdepositionen in deutschen FFH-Gebieten 2005 
zentration von Stoffen dar, die sich an der äußeren Hülle des Lebewesens sammeln oder von dort in sein Inneres gelangt sind. Die äußere Exposition sind z. B. Konzentrationen von Stoffen in den Umweltkompartimenten, mit denen der Organismus Stoffe austauscht oder Stoffflüsse (-frachten), die aus diesen Umgebungsmedien an der Oberfläche des Lebewesens abgeschieden (deponiert) werden. Stoffanreicherungen in Lebewesen sind vielfach Ausgangspunkt toxikologischer Untersuchungen, in denen diejenige Stoffmenge gesucht wird, die eine physiologische Veränderung des Organismus hervorruft. Stoffanreicherungen in Organismen bergen also ein Wirkpotenzial in sich und sind von daher toxikologisch relevant. Atmosphärische Depositionen erlangen toxikologische Relevanz, wenn sie mit artspezifischen ökosystemaren Wirkschwellen, z.B. den Critical Loads, verknüpft werden (Augustin et al. 2005; Hansen et al. 2007; Lorenz und Granke 2009). Die Aussagekraft von Critical Loads bemisst sich nach der Qualität des Modells, mit dem sie berechnet wurden und nach ihrer räumlichen Auflösung (Barkman und Alveteg 2001; Spranger et al. 2001; Wuyts et al. 2009).

In dieser Arbeit wird die Exposition der in Deutschland eingerichteten Natura 2000 SCI anhand der Deposition und Akkumulation von Metallen und $\mathrm{N}$ gekennzeichnet. Es wurde gezeigt, dass sich der physikalische Zusammenhang zwischen atmosphärischer Deposition und Stoffanreicherung in statistischen Zusammenhängen spiegelt, die in Form linearer Regressionsmodelle beschrieben werden können. Die Anwendung dieser Modelle auf das räumlich dichte Moosmessnetz ermöglicht eine hinreichend räumlich differenzierte Schätzung von Stoffeinträgen in Natura-2000-FFH-Gebieten. Depositionskarten bilden eine Grundlage für die die Berechnung von Critical Loads Exceedances (Augustin et al. 2005; Hansen et al. 2007; Spranger et al. 2001). Überdies ermöglichen die Daten aus dem Moosmonitoring die Abschätzung der Stoffanreicherung in Natura-2000-FFH-Gebieten. Demnach zeigt sich ein statistisch signifikanter Rückgang der Schwermetallanreicherungen zwischen 1990 und 2000 und zwischen 2000 und 2005 ein statistisch signifikanter Anstieg. Ferner können diejenigen FFH-Gebiete Deutschlands identifiziert werden, in denen die Stickstoffanreicherung kritische Grenzwerte überschreitet. Beispiele hierfür sind das 98. Perzentil für die $\mathrm{Pb}$-Deposition $(13,73 \mathrm{~g} / \mathrm{ha} / \mathrm{a})$, für die Cd-Deposition $(0,54 \mathrm{~g} / \mathrm{ha} / \mathrm{a})$ sowie die Gesamtstickstoffdeposition $(25,6$ $\mathrm{kg} / \mathrm{ha} / \mathrm{a})$. Sowohl die Metall- als auch die Stickstoffexposition konnte für die FFH-Gebiete naturräumlich und nach Bundesländern differenziert ausgewertet werden. Das Moosmonitoring ist daher ein die Atmosphäre (Deposition) und die Biosphäre (Anreicherung in Moosen) umfassendes Programm der Langfristumweltbeobachtung. Sektorübergreifende Datenhaltungen für Depositionsdaten sind nach UMEG (2004) bislang kaum eingeführt, und es besteht ein Bedarf an raum- und medienübergreifenden Bilanzie- rungen. Über drei räumliche und administrative Ebenen - Region (z. B. Bundesland oder Naturraum/Natura-2000Gebiete), Staat (z. B. Deutschland) und Kontinent (Europa) - stellt das Moosmonitoring harmonisierte, qualitätskontrollierte Daten zur Bioakkumulation von Stoffen bereit. Eine derart breite Palette von analysierten Stoffen ist in keinem anderen Messnetz vorhanden. Zudem werden die (öko-)toxikologisch relevanten Schwermetalle und Stickstoff zur Umweltüberwachung und Wirksamkeitskontrolle umweltpolitischer Maßnahmen über ein INSPIRE ${ }^{3}$ - und PortalU ${ }^{4}$-konformes WebGIS ${ }^{5}$-Portal zugängig gemacht. Der in diesem Artikel vorgestellte Ansatz sollte für die Abschätzung der Schwermetall- und Stickstoffexposition aller SCI in Europa angewendet und mit Systemen des Biodiversitätsmonitorings (Fischer et al. 2009; Hammen et al. 2009) verknüpft werden.

Inwieweit das Moosmonitoring oder andere Messnetze wie z.B. die Depositionsmessnetze tatsächlich Hintergrundwerte erfassen, hängt zum einen von der Definition und zum anderen von der Operationalisierung des Begriffes „Hintergrundwert“ ab. Eine allgemein akzeptierte, darüber hinausgehende operationale Definition des Begriffes für atmosphärische Depositionen ist nicht etabliert (Hellmann 2001). Demgegenüber stellen Erhardt et al. (1996) ein statistisches Verfahren vor, das die „Bestimmung der gebietstypischen ,Normal- bzw. Hintergrundbelastung ${ }^{6}$ und der Schwellenwerte für den Nachweis der Immissionsbelastung“ ermöglicht. Mediane der Hintergrundwerte der CdDeposition betragen demnach für Industrie- und Siedlungsgebiete $1,1 \mathrm{~g} / \mathrm{ha} / \mathrm{a}$ und für sogenannte Außenbereiche $0,7 \mathrm{~g} /$ ha/a (UMEG 2004). Die 10. Perzentile betragen $0,4 \mathrm{~g} / \mathrm{ha} / \mathrm{a}$ für Siedlungs- und Außenbereiche, $0,5 \mathrm{~g} / \mathrm{ha} / \mathrm{a}$ für Industriegebiete. Die 90. Perzentile betragen 4,4 g/ha/a (Industrie), $2,7 \mathrm{~g} / \mathrm{ha} / \mathrm{a}$ (Siedlungen) bzw. 1,1 g/ha/a (Außenbereiche). Die Mediane der Hintergrundwerte der Pb-Deposition werden für Industriegebiete auf $61 \mathrm{~g} / \mathrm{ha} / \mathrm{a}$, für Siedlungsgebiete auf $45 \mathrm{~g} / \mathrm{ha} / \mathrm{a}$ und für Außenbereiche auf $26 \mathrm{~g} / \mathrm{ha} / \mathrm{a}$ geschätzt. Die 10. Perzentile betragen $28 \mathrm{~g} / \mathrm{ha} / \mathrm{a}, 18 \mathrm{~g} / \mathrm{ha} / \mathrm{a}$ bzw. $13 \mathrm{~g} /$ ha/a, die 90 . Perzentile $242 \mathrm{~g} / \mathrm{ha} / \mathrm{a}, 571 \mathrm{~g} / \mathrm{ha} / \mathrm{a}$ bzw. 72. Alle diese Werte werden als vorläufige, grobe Hintergrundwerte für den Raum Schweiz, Österreich und Süddeutschland bezeichnet und beziehen sich auf die Jahre 2000 bis 2003 (UMEG 2004). Die in dieser Veröffentlichung vorgestellten, auf der Basis der Kombination von modellierten Depositionen und Moosakkumulationen berechneten Depositionen für $\mathrm{Cd}$ und $\mathrm{Pb}$ in $\mathrm{FFH}-G e b i e t e n$ liegen insgesamt deutlich unter den von UMEG (2004) veröffentlichten Werten für

\footnotetext{
${ }^{3}$ Infrastructure for Spatial Information in the European Community (INSPIRE) http://inspire.jrc.ec.europa.eu/.

${ }^{4}$ Umweltportal Deutschland http://www.portalu.de/.

${ }^{5}$ Geoinformationssysteme (GIS), deren Funktionen auf der Netzwerktechnologie (Internet, Intranet) basieren (gebräuchlich auch: GIS online, Internet-GIS, NetGIS, Distributed GIS).
} 
den Industrie- und Siedlungsbereich. Im Hinblick auf den Außenbereich sind die Unterschiede zwar weniger deutlich, aber doch immer noch auffällig. Ein Grund für diese Abweichungen könnte in der unterschiedlichen methodischen Herangehensweisen beider Ansätze begründet sein. So verwendet UMEG (2004) Messinformationen aus technischen Sammlern zur Ableitung der Hintergrundwerte. Die Ableitung der $\mathrm{Cd}-, \mathrm{Pb}$ - und $\mathrm{N}$-Depositionskarten beruht hingegen auf geostatistisch verallgemeinerten Flächenschätzungen der Akkumulation dieser Stoffe in Moosen (Schröder et al. $2010 a, b)$.

Einen rezeptoridentischen Vergleich mit realen Hintergrundwerten ermöglicht eine Studie aus Nordostgrönland von Zechmeister et al. (eingereicht). Hier wurden in der Umgebung der Forschungsstation Zackenberg an acht Standorten Proben der Moosarten Sphagnum olafii (Flatberg) und Aulacomnium turgidum (Wahlenberg) Schwägr. gesammelt und auf elf Metalle (inkl. $\mathrm{Cd}$ und $\mathrm{Pb}$ ) chemisch analysiert. Für die $\mathrm{Cd}-$ und $\mathrm{Pb}-$ Konzentration in den Moosen konnte sich so eine mittlere Elementkonzentration von $0,041 \mu \mathrm{g} / \mathrm{g}$ für $\mathrm{Cd}$ und $0,16 \mu \mathrm{g} / \mathrm{g}$ für $\mathrm{Pb}$ berechnen lassen. Wendet man die in Abschn. 3.2 erwähnten Regressionsfunktionen ohne Berücksichtigung der räumlich autokorrelierten Residuen nach Schröder et al. (2010b) auf diese Werte an, so ergeben diese Depositionshintergrundwerte von $0,078 \mathrm{~g} / \mathrm{ha} / \mathrm{a}$ für $\mathrm{Cd}$ bzw. $0,58 \mathrm{~g} / \mathrm{ha} / \mathrm{a}$ für $\mathrm{Pb}$. Die für FFH-Gebiete in Deutschland ermittelten durchschnittlichen Depositionen aus Abb. 2 liegen somit deutlich über diesen Hintergrundwerten. Bei diesem Vergleich muss allerdings berücksichtigt werden, dass die von Zechmeister et al. (eingereicht) beprobten Moosarten in den europaweiten Moosmonitoringkampagnen nicht $\mathrm{zu}$ den prioritären Moosarten zählen. Brown (1984) gibt für die im Nordosten Grönlands gesammelten Bult-Sphagnen eine leicht erhöhte Aufnahmekapazität gegenüber den prioritären Moosarten des europäischen Moosmonitorings an. Die tatsächlichen Hintergrundwerte bezüglich dieser Arten würden somit sogar noch etwas tiefer liegen.

Für zukünftige Arbeiten sollten die Ergebnisse aus dem Moosmonitoring in das von Gauger et al. (2008a) erarbeitete Depositionsmodellierungskonzept eingepasst werden. Neben empirisch untermauerten Modellen für die Trockendeposition und die okkulte Deposition verwenden Gauger et al. (2008a) auch geostatistisch abgeleitete Karten für die nasse Deposition. Grundlage letzterer Karten sind v. a. punktuelle Bulk-Messungen verschiedener Messnetze aus ganz Deutschland sowie von grenznahen Stationen benachbarter Länder (Österreich, Tschechien, Polen). Da bei diesen Daten auch die trockene Deposition mit erfasst wird, werden die Bulk-Messdaten auf Basis von empirisch ermittelten Umrechnungsfaktoren auf den nass deponierten Anteil korrigiert. Eine Anpassung dieser Vorgehensweise auf die aus den Moosmonitoringdaten abgeleiteten Depositionsra- ten sollte in weiteren Arbeiten geprüft werden. Hierdurch ließe sich die räumliche Dichte der Messdaten für die nasse Deposition signifikant erhöhen. Die Ergebnisse der geostatistischen Berechnungen würden hierdurch an Plausibilität gewinnen.

\section{Literatur}

Ahlf W, Drost W, Heise S (2009) Incorporation of metal bioavailability into regulatory frameworks - metal exposure in Water and sediment. J Soils Sediments 9:411-419

Augustin S, Bolte A, Holzhausen M, Wolf B (2005) Exceedance of critical loads of nitrogen and sulphur and its relations to forest condition. Eur J Forest Res 124:289-300

Barkman A, Alveteg M (2001) Effects of data uncertainty in the Swedish critical loads assessment for forest soils. Water Air Soil Pollut 125:133-156

Brown DH (1984) Uptake of mineral elements and their use in pollution monitoring. In: Dyer AF, Ducket JG (Hrsg) The experimental biology of bryophytes. Academic Press, London, S 55-62

BMU (Bundesministerium für Umwelt, Naturschutz und Reaktorsicherheit) (2006) Schadstoffregister PRTR. http://www.bmu.de/ luftreinhaltung/downloads/doc/36906.php. Zugriff: 1. Februar 2010

BMU (2007) Nationale Strategie zur biologischen Vielfalt. http:// www.bmu.de/naturschutz_biologische_vielfalt/downloads/ doc/40333.php. Zugriff: 1 Februar 2010

Doyle U, Heiß C (2009) Bewertung von diffusen Stoffeinträgen im Rahmen der nationalen Biodiversitätsstrategie. Renaissance der integrierten Umweltbeobachtung. Umweltwiss Schadst Forsch 21:539-548

Erhardt W, Höpker K, Fischer I (1996) Verfahren zur Auswertung von Ergebnissen standardisierter Graskulturen und Schwellenwerte zur Bewertung immissionsbedingter Stoffanreicherungen. Umweltwiss Schadstoff-Forsch 8(4):237-240

Erisman JW, de Vries W (2000) Nitrogen deposition and effects on European forests. Environ Rev 8(2):65-93

Fischer R, Granke O, Chirici G, Meyer P, Seidling W, Stofer S, Corona P, Marchetti M, Travaglini D (2009) Background, main results and conclusions from a test phase for biodiversity assessments on intensive forest monitoring plots in Europe. iForest 2:67-74

Forster E-M, Matthies M, Brüggemann R (1993) Optimierung eines Bioindikator-Meßnetzes mit geostatistischen Methoden und einem geografischen Informationssystem. Umweltwiss Schadst Forsch 5:286-294

Förstner U (2009) Sediments and priority substances in river basins new directive 2008/105/EC; sediment issues in management plans. J Soils Sediments 9:89-93

Galloway JN, Townsend AR, Erisman JW, Bekunda M, Cai Z, Freney JR, Martinelli LA, Seitzinger SP, Sutton MA (2008) Transformation of the nitrogen cycle: Recent trends, questions, and potential solutions. Science 320(5878):889-892

Gauger T (2005) Nationale Luftreinhaltestrategie - Umsetzung von EU-Anforderungen; Teilvorhaben 02: Aufbereitung, Nutzung und Weiterentwicklung nationaler, hochauflösender Datensätze zu Konzentrationen und Depositionen von Luftschadstoffen. Forschungsvorhaben im Auftrag des BMU/UBA, FE-Nr. 20343 257/02. FAL-AOE, Braunschweig

Gauger T, Anshelm F (2002) Mapping of ecosystem specific long-term trends in deposition loads and concentrations of air pollutants in Germany and their comparison with Critical Loads and Critical Levels. Part 2: Mapping Critical Levels exceedances. Research Project 29942210 on behalf of Federal Environmental Agency. Final Report, Berlin Stuttgart 
Gauger T, Anshelm F, Köble R (2000a) Kritische LuftschadstoffKonzentrationen und Eintragsraten sowie ihre Überschreitung für Wald und Agrarökosysteme sowie naturnahe waldfreie Ökosysteme. Forschungsvorhaben im Auftrag des BMU/UBA, FE-Nr. 297 85 079, Teil 1: Deposition Loads. Institut für Navigation, Universität Stuttgart

Gauger T, Anshelm F, Köble R (2000b) Kritische LuftschadstoffKonzentrationen und Eintragsraten sowie ihre Überschreitung für Wald und Agrarökosysteme sowie naturnahe waldfreie Ökosysteme. Forschungsvorhaben im Auftrag des BMU/UBA, FE-Nr. 297 85 079, Teil 2: Critical Levels. Institut für Navigation, Universität Stuttgart

Gauger T, Anshelm F, Schuster H, Draaijers GPJ, Bleeker A, Erisman JW, Vermeulen AT, Nagel HD (2002) Mapping of ecosystem specific long-term trends in deposition loads and concentrations of air pollutants in Germany and their comparison with Critical Loads and Critical Levels. Part 1: Deposition Loads 1990-1999. Research Project 29942210 on behalf of Federal Environmental Agency. Final Report, Berlin Stuttgart

Gauger T, Haenel H-D, Rösemann C, Dämmgen U, Bleeker A, Erisman JW, Vermeulen AT, Schaap M, Timmermanns RMA, Builtjes PJH, Duyzer JH (2008a) National Implementation of the UNECE Convention on Long-range Transboundary Air Pollution (Effects). Part 1: Deposition Loads: Methods, modelling and mapping results, trends. UBA-Texte 38/08

Gauger T, Haenel H-D, Rösemann C, Nagel H-D, Becker R, Kraft P, Schlutow A, Schütze G, Weigelt-Kirchner R, Anshelm F (2008b) Nationale Umsetzung der EU-NEC-Richtlinie/CAFE-Strategie und UNECE Luftreinhaltekonvention. Teil 2: Wirkungen und Risiokoabschätzungen, Critical Loads, Biodiversität, Dynamische Modellierung, Critical Levels Überschreitungen, Materialkorrosion. UBA Texte 39/08

Genßler L, Rademacher J, Rammert U (2001) Arbeitskreis der Landesanstalten und -ämter. Konzeption der künftigen Aufgabenbereiche. Umweltwiss Schadst Forsch 13:1-4

Gundersen P, Rasmussen L (1990) Nitrification in forest soils: Effects from nitrogen deposition on soil acidification and aluminium release. Rev Environ Contam Toxicol 113:1-45

Hammen VC, Biesmeijer JC, Bommarco R, Budrys E, Christensen TR, Fronzek S, Grabaum R, Jaksic P, Klotz S, Kramarz P, Kröel-Dulay G, Kühn I, Mirtl M, Moora M, Petanidou T, Pino J, Potts SG, Rortais A, Schulze CH, Steffan-Dewenter I, Stout J, Szentgyörgyi H, Vighi M, Vujic A, Westphal C, Wolf T, Zavala G, Zobel M, Settele J, Kunin WE (2009) Establishment of a cross-European field site network in the ALARM project for assessing large-scale changes in biodiversity. Environ Monit Assess. doi:10.1007/ s10661-009-0896-7

Hansen K, Vesterdahl L, Bastrup-Birk A, Bille-Hansen J (2007) Are indicators for critical loads exceedance related to forest condition? Water Air Soil Pollut 183:293-308

Harmens H, Norris D et al. (2008) Spatial and temporal trends in heavy metal accumulation in mosses in Europe (1990-2005). Programme Coordination Centre for ICP Vegetation, Environment Centre Wales, Bangor, UK

Harmens H, Cooper D, Norris D, Schröder W, Pesch R, Holy M, Fagerli H (2009) Comparison of modelled nitrogen deposition and nitrogen concentrations in mosses. In: Benedictow A, Fagerli, H, Gauss, M, Jonson J E, Nyíri Á, Simpson D, Tsyro S, Valdebento A, Valiyaveetil S, Wind P, Aas W, Hjelbrekke A-G, Mareckova $K$, Wankmüller R, Harmens H, Cooper D, Norris D, Schröder W, Pesch R, Holy M (Hrsg) Transboundary acidification, eutrophication and ground level ozone in Europe in 2007. EMEP status report 2009. EMEP Report 1/2009, Oslo

Hellmann H (2001) Definition der Hintergrund- bzw. BackgroundKonzentration - eine Übersicht. Acta Hydrochim Hydrobiol 29(6/7):391-398
Herpin U, Siewers U, Markert B, Rosolen V, Breulmann G, Bernoux M (2004) Second German heavy metal survey by means of mosses, and comparison of the first and second approach in Germany and other European countries. Env Sci Pollut Res 11:57-66

Knappe F, Möhler S, Ostermayer A, Lazar S, Kaufmann C (2008) Vergleichende Auswertung von Stoffeinträgen in Böden über verschiedene Eintragspfade. UBA-Texte 36/08, Dessau

Kostka-Rick R, Leffler US, Markert B, Herpin U, Lusche M, Lehrke J (2001) Biomonitoring zur wirkungsbezogenen Ermittlung der Schadstoffbelastung in terrestrischen Ökosystemen. Konzeption, Durchführung und Beurteilungsmaßstäbe im Rahmen von Genehmigungsverfahren. Umweltwiss Schadst Forsch $12: 5-12$

Lorenz M, Granke O (2009) Deposition measurements and critical loads calculations: monitoring data, results and perspective. iForest 2:11-14

MERAG (2007) Incorporation of bioavailability for water, soils and sediments. Metals Risk Assessment Guidance (MERAG) Fact Sheet 05. ICMM, London. http://www.euras.be/assets/files/MERAG/ MERAG\%20FS\%2005\%20Jan\%2007.pdf. Zugriff: 1 Februar 2010

Mohaupt-Jahr B, Küchler-Krischun J (2008) Die nationale Strategie zur biologischen Vielfalt. Umweltwiss Schadst Forsch 20:104 111

Mohr K (1999) Passives Monitoring von Stickstoffeinträgen in Kiefernforsten mit dem Rotstengelmoos Pleurozium schreberi (Brid) Mitt Umweltwiss Schadst Forsch 11:267-274

Mohr K (2007) Biomonitoring von Stickstoffimmissionen. Möglichkeiten und Grenzen von Bioindikationsverfahren. Umweltwiss Schadst Forsch 19(4):255-264

Mohr K, Holy M, Pesch R, Schröder W (2009) Bioakkumulation von Metallen und Stickstoff zwischen 1990 und 2005 in Niedersachsen. Umweltwiss Schadstoff-Forsch 21:459-464

O'Connor P (2009) Monitoring - Quality assurance and quality control. Government of South Australia. South Australian MurrayDarlin Basin. Natural Resources Management Board http:// www.samdbnrm.sa.gov.au/Portals/7/Monitoring\%20Quality\%20 Assurance $\% 20$ and $\% 20$ Quality\%20Control\%20(C).pdf. Zugriff: 25 November 2009

OSPAR (Convention for the Protection of the Marine Environment of the North-East Atlantic) (2007) OSPAR List of chemicals for priority action (update 2007). http://www.ospar.org/documents/dbase/decrecs/agreements/04-12e List $\% 20$ of $\% 20$ Chemicals $\% 20$ for\%20Priority\%20action.doc. Zugriff: 1 Februar 2010

Pesch R, Schröder W, Dieffenbach-Fries H, Genßler L, Kleppin L (2008) Optimierung des Moos-Monitoring-Messnetzes in Deutschland. Umweltwiss Schadst Forsch 20:49-61

Pitcairn C, Leith I, Sheppard L, Sutton M, Fowler D, Munro R, Tang S, Wilson D (1998) The relationship between nitrogen deposition species composition and foliar nitrogen concentrations in woodland flora. Environ Pollut 102(S1):41-48

Pitcairn C, Fowler D, Leith I, Sheppard L, Tang S, Sutton M, Famulari D (2006) Diagnostic indicators of elevated nitrogen deposition. Environ Pollut 144:941-950

Schröder W, Pesch R (2005) Time series of metals in mosses and their correlation with selected sampling site-specific and ecoregional characteristics in Germany. Env Sci Pollut Res 12:159-167

Schröder W, Englert C, Pesch R, Zechmeister HG, Thöni L, Suchara I, Maňkovská B, Jeran Z, Harmens H, Grodzinska K, Alber R (2008a) Metallakkumulation in Moosen: Standörtliche und regionale Randbedingungen des Biomonitoring von Luftverunreinigungen. Umweltwiss Schadst Forsch 20:120-132

Schröder W, Hornsmann I, Pesch R, Schmidt G, Fränzle S, Wünschmann S, Heidenreich H, Markert B (2008b) Moos-Monitoring als Spiegel der Landnutzung? Stickstoff- und Metallakkumulation in Moosen zweier Regionen Mitteleuropas. Umweltwiss Schadst Forsch 20:62-74 
Schröder W, Pesch R, Matter Y, Dieffenbach-Fries H, Genßler L (2009) Trend der Schwermetall-Bioakkumulation 1990 bis 2005 in Deutschland. Umweltwiss Schadst Forsch 21:549-574

Schröder W, Holy M, Pesch R, Harmens H, Fagerli H (2010a) Kartierung der Hintergrundwerte atmosphärischer Stickstoff-Gesamtdepositionen in Deutschland anhand von Daten des EMEPMessnetzes und des ICP Vegetation Moos-Monitoring 2005. Umweltwiss Schadst Forsch (eingereicht)

Schröder W, Holy M, Pesch R, Harmens H, Ilyin I (2010b) Kartierung atmosphärischer Depositionen von Blei und Cadmium in Deutschland mit Daten aus dem EMEP-Messnetz und dem Europaweiten Moos-Monitoring 2005 Umweltwiss Schadst Forsch (eingereicht)

Selck H, Forbes VE (2004) The relative importance of water and diet for uptake and subcellular distribution of cadmium in the depositfeeding polychaete Capitella sp. I. Mar Environ Res 57:261-279

Slanina S, Wayne D (2008) Air pollution emissions. In: Cleveland CJ (Hrsg) Encyclopedia of Earth. First published in the Encyclopedia of Earth. http://www.eoearth.org/article/Air_pollution_emissions. Zugriff: 21. August 2009

Solga A, Burkhardt J, Zechmeister HG, Frahm J-P (2005) Nitrogen content, $15 \mathrm{~N}$ natural abundance and biomass of the two pleurocarpous mosses Pleurozium schreberi (Brid.) Mitt., and Scleropodium purum (Hedw.) Limpr. in relation to atmospheric nitrogen deposition. Environ Pollut 134:465-473

Spranger T, Kunze F, Gauger T, Nagel D, Bleeker A, Draaijers G (2001) Critical loads exceedances in Germany and their dependence on the scale of input data. Water Air Soil Pollut Focus 1:335-351

Suchara I, Florek M, Godzik B, Mankovska B, Rabnecz G, Sucharová J, Tuba Z, Kapusta P (2007) Mapping of main sources of pollutants and their transport in the Visegrad space. Part I: Eight toxic metals. Expert group on biomonitoring the atmospheric deposition loads in the Visegrad countries. Pruhonice

Sucharová J, Suchara I, Holá M (2008) Contents of 37 elements in moss and their temporal and spatial trends in the Czech Republic during the last 15 years. Fourth Czech bio-monitoring survey pursued in the framework of the international programme UNECE ICP Vegetation 2005/2006. Silva Tarouca Research Institute for Landscape and Ornamental Gardening. Publ. Res. Inst. Pruhonice

UBA (Umweltbundesamt) (2008) Integrierte Strategie zur Minderung von Stickstoffemissionen. Dessau

UBA (Umweltbundesamt) (2010) Workshop zur Modellierung von Schadstoffeinträgen und ihren Wirkungen auf Ökosysteme am
8. 12.2009 im Umweltbundesamt. http://www.umweltbundesamt. de/luft/infos/veranstaltungen/ws_mapesi.htm. Zugriff: 26. Januar 2010

UMEG (Zentrum für Umweltmessungen, Umwelterhebungen und Gerätesicherheit Baden-Württemberg) (2004) Hintergrundwerte für atmosphärische Depositionen. Stand 3/2004. Bezug ab Juni 2009: http://www.fachdokumente.lubw.baden-wuerttemberg.de/servlet/ is/91766/U12-U46-N04.pdf?command=downloadContent\&filen ame $=$ U12-U46-N04.pdf\&FIS=91063. Zugriff: 1 Februar 2010

UNECE (United Nations Economic Commission for Europe) (1979) Convention on Long-range Transboundary Air Pollution (LRTAP). http://www.umweltbundesamt.de/umweltbeobachtung/uid/unece/ index.htm. Zugriff: 1 Februar 2010

UNECE/CLRTAP (2005) Heavy metals in European mosses: 2005/2006 survey. Monitoring manual. ICP Vegetation Coordination Centre, Centre for Ecology and Hydrology, Bangor, UK

Wappelhorst O, Korhammer S, Leffler US, Markert B (2000) Ein Moosbiomonitoring zur Ermittlung atmosphärischer Elementeinträge in die Euroregion Neiße (D, PL, CZ) Umweltwiss Schadst Forsch 12(4):191-200

Whitfield C, Strachan I (2009) Assessing nitrogen deposition impacts on conservation status. Background paper for the Nitrogen Deposition \& Natura 2000 Workshop, 18.-20. Mai 2009, Brussels. http://cost729.ceh.ac.uk/n2kworkshop/documents. Zugriff: 29. Oktober 2009

Worms I, Simon DF, Hassler CS, Wilkinson KJ (2006) Bioavailability of trace metals to aquatic microorganisms, importance of chemical, biological and physical processes on biouptake. Biochimie $88: 1721-1731$

Wuyts K, De Schrijver A, Verheyen K (2009) The importance of forest type when incorporating forest edge deposition in the evaluation of critical load exceedance. iForest 2:43-45

Zechmeister HG, Grodzinska K, Szarek-Lukaszewska G (2003) Bryophytes. In: Markert BA, Breure AM, Zechmeister HG (Hrsg) Bioindicators \& Biomonitors - Principles, Concepts and Applications. Elsevier, Amsterdam

Zechmeister HG, Dullinger S, Hohenwallner D, Riss A, Hanus-Illnar A, Scharf S (2006) Pilot study on road traffic emissions (PAHs, heavy metals) measured by using mosses in a tunnel experiment in Vienna, Austria. Environ Sci Pollut Res 13:398-405

Zechmeister HG, Dullinger S, Köllensperger G, Ertl S, Lettner C, Reiter K (2010) Do metal concentrations in moss from the Zackenberg area, Northeast Greenland provide a baseline for monitoring? Environ Sci Pollut Res (accepted) 